\title{
Effects of $\mathrm{PM}_{2.5}$ and its constituents on hemoglobin during the third trimester in pregnant women
}

 \\ Leqian Guo ${ }^{1} \cdot$ Mei Chun Chung ${ }^{4}$
}

Received: 8 September 2021 / Accepted: 12 January 2022 / Published online: 20 January 2022

(c) The Author(s) 2022

\begin{abstract}
Anemia has been a public health issue evoking global concern, and the low hemoglobin $(\mathrm{Hb})$ concentration links to adverse pregnancy outcomes. However, the associations of $\mathrm{PM}_{2.5}$ and its constituents with $\mathrm{Hb}$ and anemia in pregnant women remain unclear. In this retrospective birth cohort study, 7932 pregnant women who delivered in the First Affiliated Hospital of Xi'an Jiaotong University from 2015 to 2018 were included. The $\mathrm{Hb}$ during the third trimester in pregnant women was assessed before delivery. $\mathrm{PM}_{2.5}$ and its constituents $\left(\mathrm{BC}, \mathrm{NH}_{4}{ }^{+}, \mathrm{NO}_{3}{ }^{-}, \mathrm{OM}, \mathrm{SO}_{4}{ }^{2-}\right.$, and Dust) during pregnancy were retrieved from the V4.CH.03 product constructed by the Atmospheric Composition Analysis Group. Generalized linear regression model was applied to investigate the effects of $\mathrm{PM}_{2.5}$ and its constituents on $\mathrm{Hb}$ and anemia during the third trimester in pregnant women. The means and standard deviations of $\mathrm{PM}_{2.5}$, $\mathrm{BC}, \mathrm{NH}_{4}{ }^{+}, \mathrm{NO}_{3}{ }^{-}, \mathrm{OM}, \mathrm{SO}_{4}{ }^{2-}$, and Dust were 69.56 (15.24), 10.02 (2.72), 8.11 (1.77), 14.96 (5.42), 15.36 (4.11), 10.08 (1.20), and $10.98(1.85) \mu \mathrm{g} / \mathrm{m}^{3}$, respectively. Per IQR increase $\left(\mu \mathrm{g} / \mathrm{m}^{3}\right)$ of $\mathrm{PM}_{2.5}, \mathrm{BC}, \mathrm{NO}_{3}{ }^{-}$, and $\mathrm{OM}$ linked to $-0.75(-1.50,-0.01),-0.85$ $(-1.65,-0.04),-0.79(-1.56,-0.03)$, and $-0.73(-1.44,-0.03) \mathrm{g} / \mathrm{L}$ decrease of $\mathrm{Hb}$ during the third trimester in multiparous pregnant women, but not for $\mathrm{NH}_{4}{ }^{+}, \mathrm{SO}_{4}{ }^{2-}$, Dust, and primiparous pregnant women. $\mathrm{PM}_{2.5}$ and its constituents had no significant association with anemia, except for Dust (OR: 0.90, 95\% CI: 0.82, 0.99, per IQR increase) in primiparous pregnant women. Besides, $\mathrm{SO}^{2-}$ was of lag effects on $\mathrm{Hb}$ and anemia in multiparous pregnant women. Moreover, non-linear associations were found among $\mathrm{PM}_{2.5}$ and its constituents, $\mathrm{Hb}$, and anemia. Therefore, exposure to $\mathrm{PM}_{2.5}$ and some constituents of $\mathrm{PM}_{2.5}$ was associated with reduced $\mathrm{Hb}$ level during the third trimester in multiparous pregnant women. Related departments and pregnant women should take targeted actions to eliminate the detrimental effects of $\mathrm{PM}_{2.5}$ and its constituents on pregnancy outcomes.
\end{abstract}

Keywords $\mathrm{PM}_{2.5} \cdot$ Constituents of $\mathrm{PM}_{2.5} \cdot$ Hemoglobin $\cdot$ Anemia $\cdot$ Pregnant women $\cdot$ Third trimester

Responsible editor: Ludek Blaha.

Guilan Xie and Jie Yue contributed equally.

Wenfang Yang

wenfang.yang@xjtu.edu.cn

1 Department of Obstetrics and Gynecology, Maternal \& Child Health Center, The First Affiliated Hospital of Xi'an Jiaotong University, No. 277, West Yanta Road, Shaanxi Province 710061 Xi'an, People's Republic of China

2 School of Public Health, Xi' an Jiaotong University Health Science Center, Xi' an, Shaanxi Province, People's Republic of China

3 Department of Pediatrics, The First Affiliated Hospital of Xi'an Jiaotong University, Xi'an, Shaanxi Province, People's Republic of China

4 Division of Nutrition Epidemiology and Data Science, Friedman School of Nutrition Science and Policy, Tufts University, Boston, Massachusetts, USA

\section{Introduction}

Nutritional status during pregnancy not only has influence on the health of pregnant women, but also the growth and development of infant. The Developmental Origin of Health and Disease (DOHaD) concept holds that maternal nutritional status links to birth outcomes of infants and the risks of chronic diseases in adulthood via epigenetic regulation, and the health impacts even remain in multiple generations (Gluckman et al. 2016). Hemoglobin (Hb), which plays a role in oxygen transmission and exchange, is one of the proxies that mirror the nutritional status. Anemia is characterized by the decreased concentration of $\mathrm{Hb}$, which could lead to placental dysfunction through influencing the perfusion of placenta and restricting the oxygen exchange between pregnant women and fetus, so as to result in adverse pregnancy outcomes. Previous studies 
reported that the lower concentration of $\mathrm{Hb}$ in pregnant women might increase the risks of gestational hypertension, postpartum hemorrhage, perinatal mortality, preterm birth, low birth weight, and small for gestational age (Ali et al. 2020; Jung et al. 2019; Young et al. 2019).

Anemia has been a public health issue evoking global concern. It was estimated that the prevalence of anemia for pregnant women in the world was $38.2 \%$ (WHO 2015). Anemia is attributed to many factors, such as reproductive history, socioeconomic status, and environment (Çelik Kavak \& Kavak 2017; Honda et al. 2017; Ullah et al. 2019). The dominant category of anemia in pregnant women is iron deficiency anemia. $\mathrm{PM}_{2.5}$ is a complex object containing several constituents with different toxicities. A growing body of epidemiologic studies confirmed that $\mathrm{PM}_{2.5}$ had detrimental health impacts on pregnant women and fetuses (Ghassabian et al. 2019; Shang et al. 2019; Xie et al. 2021). $\mathrm{PM}_{2.5}$ can be inhaled into alveoli and enter circulatory system by lung ventilation, and then induce inflammatory stress, immune response, and alteration of iron homeostasis, ultimately change Hb level (Badman \& Jaffé 1996; Ganz 2019; Seaton et al. 1999; Weiss et al. 2019).

Although there are some researches investigating the association of $\mathrm{PM}_{2.5}$ with $\mathrm{Hb}$ and anemia, most of them focus on the elderly, adults, and children (Elbarbary et al. 2020; Honda et al. 2017; Mehta et al. 2021; Morales-Ancajima et al. 2019; Wang et al. 2021), and few focus on pregnant women (Liao et al. 2019). Meanwhile, some studies verified the negative associations of $\mathrm{PM}_{2.5}$ with $\mathrm{Hb}$ and anemia (Elbarbary et al. 2020; Honda et al. 2017; Mehta et al. 2021; Morales-Ancajima et al. 2019), but other studies found the positive associations (Liao et al. 2019; Wang et al. 2021). In addition, there is a knowledge gap in the associations of constituents of $\mathrm{PM}_{2.5}$ with $\mathrm{Hb}$ and anemia.

To address the gap, we were calculated to figure out the effects of $\mathrm{PM}_{2.5}$ and its constituents on $\mathrm{Hb}$ and anemia during the third trimester in pregnant women. It would effectively assist targeted regulatory control and individual protection, so as to minimize the detrimental effects of $\mathrm{PM}_{2.5}$ and its constituents on pregnant outcomes.

\section{Methods}

\section{Study population}

In this retrospective birth cohort study, Chinese pregnant women who delivered in the First Affiliated Hospital of Xi'an Jiaotong University from 2015 to 2018 and lived in Xi'an, China, during pregnancy were included. All information of pregnant women was retrieved from medical record. The excluded criteria contained (1) aged younger than 20 years or older than 45 years old, because the legal minimum marriage age for women in China was 20 years old (Hare-Mustin 1982) and it was very hard to conceive naturally for women who were older than 45 years old owing to the declined fertility ability (Forman et al. 2011); (2) with kidney diseases; (3) without results of blood examination about the concentration of $\mathrm{Hb}$ before delivery; and (4) gestational age was less than 28 weeks when doing the blood examination. The flowchart of participants' selection was shown in Fig. S1. Basic characteristics of the included and excluded pregnant women were of no significant differences, except for gestational diabetes (Table S1). Locations of residential address of pregnant women and hospital in the birth cohort were visualized in Fig. S2. This study was approved by the Medical Ethics Committee of the First Affiliated Hospital of Xi' an Jiaotong University (No. XJTU1AF2020LSK-261).

Gestational age was calculated by subtracting last menstrual period from the date of blood examination before delivery. Sociodemographic information, including age (years old), gestational age (weeks), gestational weight gain (GWG) (kilogram), ethnicity (Han and minorities), educational level $(\leq 9,10-12$, and $>12$ years old), occupation (farmer, worker, others, and none), gestational hypertensive disorders (yes or no), and gestational diabetes (yes or no), was collected by qualified obstetricians, midwives, and nurses. GDP and population density were extracted from the raster images of Resource and Environment Science and Data Center (https://www.resdc.cn/) and WorldPop (https:// www.worldpop.org/) with $1000 \mathrm{~m}$ buffer based on the resident addresses of pregnant women through QGIS 3.14 (https://www.qgis.org/en/site/). GDP and population density were the region-level data for the region where pregnant women lived during pregnancy, and they were divided into the low and high groups according to medians. Generally, Xi' an conducted heat warning from November to March. Considering burning coal for heat warning was the main source of $\mathrm{PM}_{2.5}$ and its constituent, and $\mathrm{Hb}$ level varied with seasons, season of blood examination was categorized as warm season (from April to October) and cold season (from November to March). Directed acyclic graph for $\mathrm{PM}_{2.5}$ and its constituents, $\mathrm{Hb}$ and anemia, and covariables was shown in Fig. S3.

\section{Assessment of $\mathrm{Hb}$ and anemia}

Venous blood of $2 \mathrm{~mL}$ was collected into the tube added with ethylenediaminetetraacetic acid when pregnant women were admitted into the hospital before delivery. The blood samples were mixed upside down and sent to the standard clinical laboratory within $2 \mathrm{~h}$ to assess the concentration of $\mathrm{Hb}$. And the blood examination was done by the Sysmex XN-3000 automated hematology analyzer (Sysmex Corporation, Kobe, Japan). The diagnostic standard of anemia for 
pregnant women was that concentration of $\mathrm{Hb}$ was lower than $110 \mathrm{~g} / \mathrm{L}$ (WHO 2011).

\section{Assessment of $P M_{2.5}$ and its constituents}

The month-level data of $\mathrm{PM}_{2.5}$ and its constituents from 2014 to 2018 were gained from the monthly gridded dataset of V4.CH.03 $\left(R^{2}=0.71, \mathrm{RMSE}=9.3 \mu \mathrm{g} / \mathrm{m}^{3}\right)$ with a resolution of $0.01^{\circ} \times 0.01^{\circ}$ and constructed by the Atmospheric Composition Analysis Group (https://sites.wustl.edu/acag/). The concentrations of $\mathrm{PM}_{2.5}$ and its constituents were estimated based on the satellite remote sensing data and calibrated with the ground monitoring data (Hammer et al. 2020; van Donkelaar et al. 2021, 2019). In this study, the constituents of $\mathrm{PM}_{2.5}$ included black carbon (BC), ammonium $\left(\mathrm{NH}_{4}{ }^{+}\right)$, nitrate $\left(\mathrm{NO}_{3}{ }^{-}\right)$, organic matter $(\mathrm{OM})$, sulfate $\left(\mathrm{SO}_{4}{ }^{2-}\right)$, and mineral dust (Dust). $\mathrm{PM}_{2.5}$ and its constituents for each pregnant woman were extracted based on the longitude and latitude corresponding to the resident address of pregnant woman with $1000 \mathrm{~m}$ buffer through QGIS 3.14. And the mean concentrations of $\mathrm{PM}_{2.5}$ and its constituents during the period between last menstrual period and the date of blood examination before delivery for each pregnant woman were calculated through time-weighted method (Lin et al. 2020; Zhao et al. 2020).

\section{Statistical analysis}

Mean and standard deviation were conducted to display the distributions of normal-distributed continuous variables. Median and interquartile range (IQR) were used to describe the skewed-distributed continuous variables. Frequency and proportion were used to describe the category variables. Student's $t$-test and chi-square analysis were adopted to identify differences between the primiparous and multiparous women. Spearman correlation was deployed to find the correlations of $\mathrm{PM}_{2.5}$ and its constituents. Generalized linear regression model was applied to investigate the effects of $\mathrm{PM}_{2.5}$ and its constituents on $\mathrm{Hb}$ and anemia during the third trimester in pregnant women. $\mathrm{PM}_{2.5}$ and its constituents were included as independent variables in the one pollution model, respectively. Besides, as red blood cells updated every 120 days, the lag effects of $\mathrm{PM}_{2.5}$ and its constituents on $\mathrm{Hb}$ and anemia were investigated by the moving average concentrations of $\mathrm{PM}_{2.5}$ and its constituents on the day of blood examination and 1, 2, 3, 4 months prior to blood examination (from lag01 to lag04 months). Furthermore, restricted cubic spline was applied to explore the non-linear associations of $\mathrm{PM}_{2.5}$ and its constituents with $\mathrm{Hb}$ and anemia.

\section{Sensitive analysis}

First, the associations were further stratified by sociodemographic variables and the modifications of sociodemographic variables were assessed with the multiplicative terms. In the stratified analysis, age, gestational age, and GWG were divided into subgroups. Second, the associations of ground monitor based $\mathrm{PM}_{2.5}$ with $\mathrm{Hb}$ and anemia were further analyzed. The $\mathrm{PM}_{2.5}$ data of 13 ground monitoring stations in Xi' an was collected from the Ministry of Ecology and Environment of the People's Republic of China (http:// www.mee.gov.cn/). And the ground monitor based $\mathrm{PM}_{2.5}$ exposure was assigned according to the residential addresses of pregnant women by inverse distance weighting method.

Package of "rms" for R 3.6.1 (https://www.r-project. org/) was applied and $p<0.05$ was considered statistically significant.

\section{Results}

\section{Baseline characteristics}

A total of 7932 pregnant women were included in this study, among which there were 5323 primiparous pregnant women and 2609 multiparous pregnant women. Baseline characteristics of the birth cohort were depicted in Table 1. Mean age of primiparous pregnant women was $28.78 \pm 3.25$ years old, and $32.77 \pm 3.75$ years old for multiparous pregnant women. Mean gestational age for primiparous and multiparous pregnant women were $39.00 \pm 1.73$ weeks and $38.35 \pm 1.89$ weeks, respectively. Mean GWG for primiparous and multiparous pregnant women were $15.70 \pm 4.53 \mathrm{~kg}$ and $14.46 \pm 4.48 \mathrm{~kg}$, respectively. Mean $\mathrm{Hb}$ concentration for primiparous and multiparous pregnant women were $119.53 \pm 13.12 \mathrm{~g} / \mathrm{L}$ and $116.16 \pm 13.90 \mathrm{~g} / \mathrm{L}$. The prevalence of anemia was $22.17 \%$ and $29.24 \%$ for primiparous and multiparous pregnant women. The differences of age, gestational age, GWG, educational level, occupation, gestational diabetes, concentration of $\mathrm{Hb}$, and anemia between primiparous and multiparous pregnant women were statistically significant $(p<0.05)$.

\section{Distributions of $P M_{2.5}$ and its constituents}

The distributions of $\mathrm{PM}_{2.5}$ and its constituents were shown in Table 2. For total pregnant women, the means and standard deviations of $\mathrm{PM}_{2.5}, \mathrm{BC}, \mathrm{NH}_{4}{ }^{+}, \mathrm{NO}_{3}{ }^{-}, \mathrm{OM}, \mathrm{SO}_{4}{ }^{2-}$, and Dust were 69.56 (15.24), 10.02 (2.72), 8.11 (1.77), 14.96 (5.42), 15.36 (4.11), 10.08 (1.20), and 10.98 (1.85) $\mu \mathrm{g} /$ $\mathrm{m}^{3}$, respectively. For primiparous pregnant women, the means and standard deviations of $\mathrm{PM}_{2.5}, \mathrm{BC}, \mathrm{NH}_{4}{ }^{+}, \mathrm{NO}_{3}{ }^{-}$, $\mathrm{OM}, \mathrm{SO}_{4}{ }^{2-}$, and Dust were 68.72 (14.60), 9.88 (2.62), 
Table 1 Baseline characteristics of the birth cohort

\begin{tabular}{|c|c|c|c|c|c|}
\hline Variables & $\begin{array}{l}\text { Total } \\
(n=7932)\end{array}$ & $\begin{array}{l}\text { Primipara } \\
(n=5323)\end{array}$ & $\begin{array}{l}\text { Multipara } \\
(n=2609)\end{array}$ & $t / \chi^{2}$ & $p$ \\
\hline Age, years old & $30.10 \pm 3.90$ & $28.78 \pm 3.25$ & $32.77 \pm 3.75$ & $-46.45^{\mathrm{a}}$ & $<0.001$ \\
\hline Gestational age, weeks & $38.79 \pm 1.81$ & $39.00 \pm 1.73$ & $38.35 \pm 1.89$ & $14.80^{\mathrm{a}}$ & $<0.001$ \\
\hline GWG & $15.29 \pm 4.55$ & $15.70 \pm 4.53$ & $14.46 \pm 4.48$ & $11.41^{\mathrm{a}}$ & $<0.001$ \\
\hline Ethnicity & & & & $2.91^{\mathrm{b}}$ & 0.09 \\
\hline Han & $7874(99.27)$ & $5278(99.15)$ & $2596(99.50)$ & & \\
\hline Minorities & $58(0.73)$ & $45(0.85)$ & $13(0.50)$ & & \\
\hline Educational level, years ${ }^{\mathrm{c}}$ & & & & $231.90^{\mathrm{b}}$ & $<0.001$ \\
\hline$\leq 9$ & $572(7.23)$ & $255(4.80)$ & $317(12.19)$ & & \\
\hline $10-12$ & $633(8.00)$ & $329(6.20)$ & $304(11.69)$ & & \\
\hline$>12$ & $6705(84.77)$ & $4726(89.00)$ & $1979(76.12)$ & & \\
\hline Occupation & & & & $71.43^{\mathrm{b}}$ & $<0.001$ \\
\hline Farmer & $231(2.91)$ & $114(2.14)$ & $117(4.48)$ & & \\
\hline Worker & $1838(23.17)$ & $1335(25.08)$ & $503(19.28)$ & & \\
\hline Others & $4937(62.24)$ & $3304(62.07)$ & $1633(62.59)$ & & \\
\hline None & $926(11.68)$ & $570(10.71)$ & $356(13.65)$ & & \\
\hline \multicolumn{4}{|c|}{ Gestational hypertensive disorders } & $2.10^{\mathrm{b}}$ & 0.15 \\
\hline Yes & $479(6.04)$ & $307(5.77)$ & $172(6.59)$ & & \\
\hline No & $7453(93.96)$ & $5016(94.23)$ & $2437(93.41)$ & & \\
\hline Gestational diabetes & & & & $16.10^{\mathrm{b}}$ & $<0.001$ \\
\hline Yes & $543(6.85)$ & $322(6.05)$ & $221(8.47)$ & & \\
\hline No & $7389(93.15)$ & $5001(93.95)$ & $2388(91.53)$ & & \\
\hline \multicolumn{6}{|l|}{ GDP } \\
\hline Low & 3965 (49.99) & $2665(50.07)$ & $1300(49.83)$ & $0.04^{\mathrm{b}}$ & 0.84 \\
\hline High & $3967(50.01)$ & $2658(49.93)$ & $1309(50.17)$ & & \\
\hline \multicolumn{6}{|l|}{ Population density } \\
\hline Low & $3966(50.00)$ & $2675(50.25)$ & $1291(49.48)$ & $0.42^{\mathrm{b}}$ & 0.52 \\
\hline High & $3966(50.00)$ & $2648(49.75)$ & $1318(50.52)$ & & \\
\hline \multicolumn{6}{|c|}{ Season of blood examination } \\
\hline Warm season & $4657(58.71)$ & $3128(58.76)$ & $1529(58.60)$ & $0.02^{\mathrm{b}}$ & 0.89 \\
\hline Cold season & $3275(41.29)$ & $2195(41.24)$ & $1080(41.40)$ & & \\
\hline $\mathrm{Hb}, \mathrm{g} / \mathrm{L}$ & $118.42 \pm 13.47$ & $119.53 \pm 13.12$ & $116.16 \pm 13.90$ & $10.36^{\mathrm{a}}$ & $<0.001$ \\
\hline Anemia & & & & $47.41^{\mathrm{b}}$ & $<0.001$ \\
\hline Yes & $1943(24.50)$ & $1180(22.17)$ & 763 (29.24) & & \\
\hline No & $5989(75.50)$ & $4143(77.83)$ & $1846(70.76)$ & & \\
\hline
\end{tabular}

Note: ${ }^{a}$ indicated Student's $t$-test, ${ }^{\mathrm{b}}$ indicated chi-square analysis, ${ }^{\mathrm{c}} n=7910$ for total pregnant women, $n=5310$ for primipara, and $n=2600$ for multipara in educational level
8.01 (1.70), 14.67 (5.20), 15.19 (3.93), 10.05 (1.21), and $10.88(1.82) \mu \mathrm{g} / \mathrm{m}^{3}$, respectively. For multiparous pregnant women, the means and standard deviations of $\mathrm{PM}_{2.5}, \mathrm{BC}$, $\mathrm{NH}_{4}{ }^{+}, \mathrm{NO}_{3}{ }^{-}, \mathrm{OM}, \mathrm{SO}_{4}{ }^{2-}$, and Dust were 71.26 (16.34), 10.31 (2.90), 8.31 (1.90), 15.57 (5.80), 15.70 (4.44), 10.16 (1.17), and $11.17(1.91) \mu \mathrm{g} / \mathrm{m}^{3}$, respectively. $\mathrm{PM}_{2.5}$ and its constituents were positively correlated both in primiparous and multiparous pregnant women, but $\mathrm{SO}_{4}{ }^{2-}$ and Dust were negatively correlated (Table S2).

\section{Linear associations of $\mathrm{PM}_{2.5}$ and its constituents with $\mathrm{Hb}$ and anemia}

The linear associations of per IQR increase $\left(\mu \mathrm{g} / \mathrm{m}^{3}\right)$ of $\mathrm{PM}_{2.5}$ and its constituents with $\mathrm{Hb}$ and anemia were provided in Fig. 1. $\mathrm{PM}_{2.5}$ and its constituents and $\mathrm{Hb}$ had no significant association in primiparous pregnant women. For multiparous pregnant women, $\mathrm{BC}$ was of the highest effect on $\mathrm{Hb}$ $(\beta:-0.85,95 \% \mathrm{CI}:-1.65,-0.04, \mathrm{~g} / \mathrm{L}$, per IQR increase $)$ 
Table 2 Distributions of $\mathrm{PM}_{2.5}$ and its constituents $\left(\mu \mathrm{g} / \mathrm{m}^{3}\right)$ of the birth cohort
Fig. 1 Linear associations of per IQR increase $\left(\mu \mathrm{g} / \mathrm{m}^{3}\right)$ of $\mathrm{PM}_{2.5}$ and its constituents with $\mathrm{Hb}$ and anemia. The linear associations of $\mathrm{PM}_{2.5}$ and its constituents with $\mathrm{Hb}$ in primipara (a) and multipara (b). The linear associations of $\mathrm{PM}_{2.5}$ and its constituents with anemia in primipara (c) and multipara (d). * indicated $p<0.05$. Adjusted for age, gestational age, GWG, ethnicity, educational level, occupation, gestational hypertensive disorders, gestational diabetes, GDP, population density, and season of blood examination

\begin{tabular}{|c|c|c|c|c|c|c|c|}
\hline Variables & Mean (SD) & IQR & Min & 25 th & 50 th & 75th & Max \\
\hline \multicolumn{8}{|l|}{ Total } \\
\hline $\mathrm{PM}_{2.5}$ & $69.56(15.24)$ & 19.37 & 8.32 & 58.72 & 67.00 & 78.09 & 125.75 \\
\hline $\mathrm{BC}$ & $10.02(2.72)$ & 3.88 & 1.06 & 7.90 & 9.77 & 11.78 & 19.65 \\
\hline $\mathrm{NH}_{4}^{+}$ & $8.11(1.77)$ & 2.04 & 1.15 & 6.87 & 7.89 & 8.91 & 14.76 \\
\hline $\mathrm{NO}_{3}^{-}$ & $14.96(5.42)$ & 7.46 & 1.07 & 10.62 & 14.28 & 18.08 & 35.26 \\
\hline $\mathrm{OM}$ & $15.36(4.11)$ & 5.00 & 1.74 & 12.37 & 15.16 & 17.37 & 29.85 \\
\hline $\mathrm{SO}_{4}{ }^{2-}$ & $10.08(1.20)$ & 1.59 & 2.12 & 9.24 & 10.00 & 10.83 & 14.00 \\
\hline Dust & $10.98(1.85)$ & 2.45 & 1.04 & 9.63 & 10.80 & 12.08 & 17.26 \\
\hline \multicolumn{8}{|l|}{ Primipara } \\
\hline $\mathrm{PM}_{2.5}$ & $68.72(14.60)$ & 18.05 & 13.15 & 58.62 & 65.93 & 76.67 & 125.47 \\
\hline $\mathrm{BC}$ & $9.88(2.62)$ & 3.69 & 1.61 & 7.87 & 9.54 & 11.56 & 19.30 \\
\hline $\mathrm{NH}_{4}^{+}$ & $8.01(1.70)$ & 1.91 & 1.52 & 6.86 & 7.83 & 8.77 & 14.35 \\
\hline $\mathrm{NO}_{3}^{-}$ & $14.67(5.20)$ & 7.05 & 2.10 & 10.53 & 13.92 & 17.58 & 34.82 \\
\hline $\mathrm{OM}$ & $15.19(3.93)$ & 4.79 & 2.56 & 12.38 & 14.97 & 17.17 & 29.51 \\
\hline $\mathrm{SO}_{4}{ }^{2-}$ & $10.05(1.21)$ & 1.58 & 2.43 & 9.21 & 9.95 & 10.79 & 14.00 \\
\hline Dust & $10.88(1.82)$ & 2.39 & 2.50 & 9.58 & 10.70 & 11.97 & 16.64 \\
\hline \multicolumn{8}{|l|}{ Multipara } \\
\hline $\mathrm{PM}_{2.5}$ & $71.26(16.34)$ & 21.85 & 8.32 & 58.99 & 69.18 & 80.84 & 125.75 \\
\hline $\mathrm{BC}$ & $10.31(2.90)$ & 4.17 & 1.06 & 8.04 & 10.29 & 12.21 & 19.65 \\
\hline $\mathrm{NH}_{4}^{+}$ & $8.31(1.90)$ & 2.30 & 1.15 & 6.93 & 8.11 & 9.23 & 14.76 \\
\hline $\mathrm{NO}_{3}^{-}$ & $15.57(5.80)$ & 8.01 & 1.07 & 10.88 & 15.14 & 18.89 & 35.26 \\
\hline $\mathrm{OM}$ & $15.70(4.44)$ & 5.50 & 1.74 & 12.36 & 15.66 & 17.86 & 29.85 \\
\hline $\mathrm{SO}_{4}^{2-}$ & $10.16(1.17)$ & 1.62 & 2.12 & 9.29 & 10.11 & 10.91 & 13.54 \\
\hline Dust & $11.17(1.91)$ & 2.51 & 1.04 & 9.79 & 10.99 & 12.30 & 17.26 \\
\hline
\end{tabular}

after adjusting for age, gestational age, GWG, ethnicity, educational level, occupation, gestational hypertensive disorders, gestational diabetes, GDP, population density, and season of blood examination, which was in line with the general knowledge that $\mathrm{BC}$ was the most harmful component of $\mathrm{PM}_{2.5}$. Following by BC, per IQR increase $\left(\mu \mathrm{g} / \mathrm{m}^{3}\right)$ of $\mathrm{NO}_{3}{ }^{-}$, $\mathrm{PM}_{2.5}$, and $\mathrm{OM}$ linked to $-0.79(-1.56,-0.03),-0.75$ $(-1.50,-0.01)$, and $-0.73(-1.44,-0.03) \mathrm{g} / \mathrm{L}$ decrease of $\mathrm{Hb}$ during the third trimester, but not for $\mathrm{NH}_{4}{ }^{+}, \mathrm{SO}_{4}{ }^{2-}$, and Dust. Likely, $\mathrm{PM}_{2.5}$ and its constituents and anemia had no significant association in primiparous pregnant women, except for Dust (OR: 0.90, 95\% CI: 0.82, 0.99, per IQR increase). However, there was no significant association of $\mathrm{PM}_{2.5}$ and its constituents with anemia being found in multiparous pregnant women.

\section{Lag effects of $\mathrm{PM}_{2.5}$ and its constituents on $\mathrm{Hb}$ and anemia}

The lag effects of $\mathrm{PM}_{2.5}$ and its constituents $\left(\mu \mathrm{g} / \mathrm{m}^{3}\right)$ on $\mathrm{Hb}$ and anemia were displayed in Table 3. The lag effects of $\mathrm{PM}_{2.5}$ and its constituents on $\mathrm{Hb}$ and anemia were not observed in primiparous pregnant women. Per IQR increase of $\mathrm{SO}_{4}{ }^{2-}$ at lag01 month decreased the $\mathrm{Hb}$ concentration

Table 3 Lag effects of $\mathrm{PM}_{2.5}$ and its constituents $\left(\mu \mathrm{g} / \mathrm{m}^{3}\right)$ on $\mathrm{Hb}$ and anemia

\begin{tabular}{|c|c|c|c|c|c|c|c|c|}
\hline \multirow[t]{2}{*}{ Variables } & \multicolumn{4}{|c|}{ Primipara $[\beta / \mathrm{OR}(95 \% \mathrm{CI})]$} & \multicolumn{4}{|c|}{ Multipara $[\beta / \mathrm{OR}(95 \% \mathrm{CI})]$} \\
\hline & Lag01 & Lag02 & Lag03 & Lag04 & Lag01 & Lag02 & Lag03 & Lag04 \\
\hline \multicolumn{9}{|l|}{$\mathrm{Hb}$} \\
\hline $\mathrm{PM}_{2.5}$ & $\begin{array}{l}-0.02 \\
(-0.27 \\
0.23)\end{array}$ & $\begin{array}{c}-0.03 \\
(-0.28 \\
0.23)\end{array}$ & $\begin{array}{c}-0.05 \\
(-0.29 \\
0.19)\end{array}$ & $\begin{array}{c}-0.08 \\
(-0.31 \\
0.16)\end{array}$ & $\begin{array}{l}-0.12(-0.56 \\
0.32)\end{array}$ & $\begin{array}{l}-0.25 \\
(-0.68,0.19)\end{array}$ & $\begin{array}{c}-0.33 \\
(-0.74 \\
0.09)\end{array}$ & $\begin{array}{l}-0.34(-0.74 \\
0.05)\end{array}$ \\
\hline $\mathrm{BC}$ & $\begin{array}{l}0.01(-0.28 \\
0.29)\end{array}$ & $\begin{array}{l}0.03(-0.25 \\
0.32)\end{array}$ & $\begin{array}{l}0.01(-0.26 \\
0.28)\end{array}$ & $\begin{array}{l}-0.04 \\
(-0.30 \\
0.22)\end{array}$ & $\begin{array}{l}-0.06(-0.54 \\
0.41)\end{array}$ & $\begin{array}{l}-0.22(-0.68 \\
0.25)\end{array}$ & $\begin{array}{c}-0.29 \\
(-0.73 \\
0.14)\end{array}$ & $\begin{array}{l}-0.30(-0.72 \\
0.12)\end{array}$ \\
\hline $\mathrm{NH}_{4}^{+}$ & $\begin{array}{c}-0.03 \\
(-0.26 \\
0.19)\end{array}$ & $\begin{array}{c}-0.01 \\
(-0.24 \\
0.23)\end{array}$ & $\begin{array}{l}-0.02 \\
(-0.25 \\
0.22)\end{array}$ & $\begin{array}{l}-0.05 \\
(-0.28 \\
0.19)\end{array}$ & $\begin{array}{l}-0.16(-0.55 \\
0.23)\end{array}$ & $\begin{array}{l}-0.28(-0.69 \\
0.13)\end{array}$ & $\begin{array}{c}-0.36 \\
(-0.76 \\
0.05)\end{array}$ & $\begin{array}{l}-0.35(-0.74 \\
0.05)\end{array}$ \\
\hline $\mathrm{NO}_{3}^{-}$ & $\begin{array}{c}-0.01 \\
(-0.29 \\
0.28)\end{array}$ & $\begin{array}{l}0.02(-0.27 \\
0.30)\end{array}$ & $\begin{array}{c}-0.01 \\
(-0.28 \\
0.26)\end{array}$ & $\begin{array}{c}-0.04 \\
(-0.30 \\
0.22)\end{array}$ & $\begin{array}{l}-0.07(-0.53 \\
0.40)\end{array}$ & $\begin{array}{l}-0.24(-0.71 \\
0.22)\end{array}$ & $\begin{array}{c}-0.33 \\
(-0.76 \\
0.11)\end{array}$ & $\begin{array}{l}-0.32(-0.73 \text {, } \\
0.10)\end{array}$ \\
\hline $\mathrm{OM}$ & $\begin{array}{l}-0.06 \\
(-0.28 \\
0.16)\end{array}$ & $\begin{array}{l}-0.02 \\
(-0.24 \\
0.20)\end{array}$ & $\begin{array}{l}-0.04 \\
(-0.25 \\
0.17)\end{array}$ & $\begin{array}{l}-0.08 \\
(-0.30 \\
0.13)\end{array}$ & $\begin{array}{c}-0.11(-0.47 \\
0.26)\end{array}$ & $\begin{array}{c}-0.21(-0.57 \\
0.15)\end{array}$ & $\begin{array}{c}-0.27 \\
(-0.62 \\
0.08)\end{array}$ & $\begin{array}{l}-0.28(-0.62 \\
0.07)\end{array}$ \\
\hline $\mathrm{SO}_{4}{ }^{2-}$ & $\begin{array}{l}-0.11 \\
(-0.35 \\
0.13)\end{array}$ & $\begin{array}{c}-0.07 \\
(-0.34 \\
0.19)\end{array}$ & $\begin{array}{c}-0.05 \\
(-0.34 \\
0.24)\end{array}$ & $\begin{array}{c}-0.07 \\
(-0.39 \\
0.25)\end{array}$ & $\begin{array}{l}-0.38 \\
\quad(-0.75,-0.01)\end{array}$ & $\begin{array}{l}-0.39(-0.81 \\
0.03)\end{array}$ & $\begin{array}{c}-0.38 \\
(-0.84 \\
0.08)\end{array}$ & $\begin{array}{l}-0.36(-0.87 \\
0.16)\end{array}$ \\
\hline Dust & $\begin{array}{l}0.03(-0.09 \\
0.16)\end{array}$ & $\begin{array}{c}-0.06 \\
(-0.20 \\
0.09)\end{array}$ & $\begin{array}{c}-0.12 \\
(-0.30 \\
0.06)\end{array}$ & $\begin{array}{l}-0.15 \\
(-0.38 \\
0.08)\end{array}$ & $\begin{array}{l}0.02(-0.17 \\
0.21)\end{array}$ & $\begin{array}{l}0.02(-0.20 \\
0.25)\end{array}$ & $\begin{array}{l}-0.05 \\
(-0.32 \\
0.23)\end{array}$ & $\begin{array}{l}-0.25(-0.60 \\
0.09)\end{array}$ \\
\hline \multicolumn{9}{|l|}{ Anemia } \\
\hline $\mathrm{PM}_{2.5}$ & $\begin{array}{l}0.97(0.93, \\
1.02)\end{array}$ & $\begin{array}{l}0.98(0.93, \\
1.03)\end{array}$ & $\begin{array}{l}1.00(0.95, \\
1.04)\end{array}$ & $\begin{array}{l}1.01(0.97, \\
1.05)\end{array}$ & $1.06(0.99,1.14)$ & $\begin{array}{l}1.06(0.98, \\
1.13)\end{array}$ & $\begin{array}{l}1.05(0.98, \\
1.12)\end{array}$ & $\begin{array}{l}1.05(0.98, \\
1.11)\end{array}$ \\
\hline $\mathrm{BC}$ & $\begin{array}{l}0.97(0.92 \\
1.02)\end{array}$ & $\begin{array}{l}0.97(0.92, \\
1.03)\end{array}$ & $\begin{array}{l}0.99(0.94, \\
1.04)\end{array}$ & $\begin{array}{l}1.01(0.96, \\
1.06)\end{array}$ & $1.03(0.96,1.11)$ & $\begin{array}{l}1.03(0.96, \\
1.12)\end{array}$ & $\begin{array}{l}1.04(0.96, \\
1.11)\end{array}$ & $\begin{array}{l}1.04(0.97, \\
1.11)\end{array}$ \\
\hline $\mathrm{NH}_{4}^{+}$ & $\begin{array}{l}0.98(0.93, \\
1.02)\end{array}$ & $\begin{array}{l}0.97(0.93, \\
1.02)\end{array}$ & $\begin{array}{l}0.99(0.95, \\
1.04)\end{array}$ & $\begin{array}{l}1.01(0.96, \\
1.05)\end{array}$ & $1.06(1.00,1.13)$ & $\begin{array}{l}1.06(0.99, \\
1.13)\end{array}$ & $\begin{array}{l}1.06(0.99, \\
1.13)\end{array}$ & $\begin{array}{l}1.05(0.98, \\
1.12)\end{array}$ \\
\hline $\mathrm{NO}_{3}^{-}$ & $\begin{array}{l}0.97(0.91, \\
1.02)\end{array}$ & $\begin{array}{l}0.97(0.92, \\
1.03)\end{array}$ & $\begin{array}{l}0.99(0.94, \\
1.05)\end{array}$ & $\begin{array}{l}1.01(0.96, \\
1.06)\end{array}$ & $1.05(0.97,1.13)$ & $\begin{array}{l}1.05(0.97, \\
1.13)\end{array}$ & $\begin{array}{l}1.04(0.97, \\
1.12)\end{array}$ & $\begin{array}{l}1.04(0.97, \\
1.11)\end{array}$ \\
\hline $\mathrm{OM}$ & $\begin{array}{l}0.99(0.95 \\
1.03)\end{array}$ & $\begin{array}{l}0.99(0.95, \\
1.03)\end{array}$ & $\begin{array}{l}1.00(0.96, \\
1.05)\end{array}$ & $\begin{array}{l}1.02(0.98, \\
1.06)\end{array}$ & $1.03(0.97,1.10)$ & $\begin{array}{c}1.03(0.97, \\
1.09)\end{array}$ & $\begin{array}{c}1.03(0.98, \\
1.09)\end{array}$ & $\begin{array}{c}1.03(0.98, \\
1.09)\end{array}$ \\
\hline $\mathrm{SO}_{4}{ }^{2-}$ & $\begin{array}{l}1.00(0.95 \\
1.04)\end{array}$ & $\begin{array}{l}0.98(0.93 \\
1.03)\end{array}$ & $\begin{array}{l}0.98(0.93 \\
1.04)\end{array}$ & $\begin{array}{l}0.99(0.94 \\
1.05)\end{array}$ & $1.09(1.03,1.16)$ & $\begin{array}{l}1.10(1.03, \\
1.17)\end{array}$ & $\begin{array}{l}1.10(1.02, \\
1.18)\end{array}$ & $\begin{array}{l}1.09(1.00, \\
1.18)\end{array}$ \\
\hline Dust & $\begin{array}{l}0.99(0.97 \\
1.01)\end{array}$ & $\begin{array}{l}1.00(0.97, \\
1.03)\end{array}$ & $\begin{array}{l}1.01(0.97, \\
1.04)\end{array}$ & $\begin{array}{l}1.00(0.96, \\
1.05)\end{array}$ & $1.01(0.98,1.05)$ & $\begin{array}{c}1.02(0.98, \\
1.05)\end{array}$ & $\begin{array}{c}1.02(0.98, \\
1.07)\end{array}$ & $\begin{array}{c}1.04(0.98, \\
1.10)\end{array}$ \\
\hline
\end{tabular}

Note: Adjusted for age, gestational age, GWG, ethnicity, educational level, occupation, gestational hypertensive disorders, gestational diabetes, GDP, population density, and season of blood examination 
$(\beta:-0.38,95 \% \mathrm{CI}:-0.75,-0.01, \mathrm{~g} / \mathrm{L})$ and per IQR increase of $\mathrm{SO}_{4}{ }^{2-}$ at lag01, lag02, and lag03 months increased the risk of anemia (OR: 1.09-1.10) in multiparous pregnant women, while lag effects of $\mathrm{PM}_{2.5}, \mathrm{BC}, \mathrm{NH}_{4}{ }^{+}, \mathrm{NO}_{3}{ }^{-}, \mathrm{OM}$, and Dust on $\mathrm{Hb}$ and anemia were not identified in other lag models for multiparous pregnant women.

\section{Non-linear associations of $P M_{2.5}$ and its constituents with $\mathrm{Hb}$ and anemia}

The non-linear associations of $\mathrm{PM}_{2.5}$ and its constituents with $\mathrm{Hb}$ and anemia were showed in Fig. 2. Summarily, there were declined trends of $\mathrm{Hb}$ with the increase of $\mathrm{PM}_{2.5}$ and its constituents both in primiparous and multiparous pregnant women and the trends slightly fluctuated at the moderate concentrations of $\mathrm{PM}_{2.5}$ and its constituents. The associations of $\mathrm{PM}_{2.5}$ and its constituents with anemia were "W" and " $\mathrm{M}$ " shapes in primiparous and multiparous pregnant women.

\section{Sensitive analysis}

Gestational age, GWG, educational level, ethnicity, occupation, gestational hypertensive disorders, gestational diabetes, population density, and season of blood examination were of modification effects on the associations of $\mathrm{PM}_{2.5}$ and its constituents with $\mathrm{Hb}$ (Table S3-9) and anemia (Table S10-16).
Additionally, our findings were robust in the further analyses with ground monitor based $\mathrm{PM}_{2.5}$ (Table S17-21, Fig. S4).

\section{Discussion}

We comprehensively explored the effects of $\mathrm{PM}_{2.5}$ and its constituents on $\mathrm{Hb}$ and anemia during the third trimester in pregnant women. Increased $\mathrm{PM}_{2.5}, \mathrm{BC}, \mathrm{NO}_{3}{ }^{-}$, and $\mathrm{OM}$ were tied to the declined $\mathrm{Hb}$ level in multiparous pregnant women, but not for $\mathrm{NH}_{4}{ }^{+}, \mathrm{SO}_{4}{ }^{2-}$, Dust, and primiparous pregnant women. The effects of $\mathrm{PM}_{2.5}$ and its constituents on $\mathrm{Hb}$ were slight, that was why the significant associations tended to be found in $\mathrm{Hb}$, but not in anemia. Besides, $\mathrm{SO}_{4}{ }^{2-}$ was of lag effect on $\mathrm{Hb}$ and anemia in multiparous pregnant women. Moreover, non-linear associations were found among $\mathrm{PM}_{2.5}$ and its constituents, $\mathrm{Hb}$, and anemia.

$\mathrm{PM}_{2.5}$ is a sophisticated mixture encompassing many constituents with different sources and toxicities. $\mathrm{PM}_{2.5}$ mainly comes from fossil fuel combustion, such as vehicle emission, power station, and factories. Each source might generate several constituents of $\mathrm{PM}_{2.5}$, and each constituent of $\mathrm{PM}_{2.5}$ might also come from several resources. The toxicities of constituents of $\mathrm{PM}_{2.5}$ not only are ascribed to their own effects, but also the interactive effects among them (Kelly \& Fussell 2012). In this study, exposure to $\mathrm{PM}_{2.5}$ reduced the $\mathrm{Hb}$ level $(\beta:-0.75,95 \% \mathrm{CI}:-1.50,-0.01, \mathrm{~g} / \mathrm{L}$, per IQR


Fig. 2 Non-linear associations of $\mathrm{PM}_{2.5}$ and its constituents with $\mathrm{Hb}$ and anemia. The non-linear associations of $\mathrm{PM}_{2.5}$ and its constituents with $\mathrm{Hb}$ in primipara (a) and multipara (b). The non-linear associations of $\mathrm{PM}_{2.5}$ and its constituents with anemia in primipara (c) and multipara (d). Adjusted for age, gestational age, GWG, ethnicity, educational level, occupation, gestational hypertensive disorders, gestational diabetes, GDP, population density, and season of blood examination 
increase of $\mathrm{PM}_{2.5}$ in multiparous pregnant women), which was in lined with the previous researches. A representative cohort study in America observed that 1-year moving average $\mathrm{PM}_{2.5}$ linked to $-0.81 \mathrm{~g} / \mathrm{dL}$ decrease of $\mathrm{Hb}$ level in older adults, and the dose-response relationship was strong (Honda et al. 2017). Another longitude study organized by $\mathrm{WHO}$ indicated that 3 -year moving average $\mathrm{PM}_{2.5}$ was negatively correlated with the concentration of $\mathrm{Hb}$ in older Chinese adults $(\beta:-0.52,95 \% \mathrm{CI}:-0.71,-0.33, \mathrm{~g} / \mathrm{L}$, per IQR increase of $\mathrm{PM}_{2.5}$ ) (Elbarbary et al. 2020). Likely, one study for children found that increased exposure to $\mathrm{PM}_{2.5}$ was tied to a slight decrease of $\mathrm{Hb}$ concentration $(\beta:-0.14$, 95\% CI: $-0.16,-0.12, \mathrm{~g} / \mathrm{L}$, per $10 \mu \mathrm{g} / \mathrm{m}^{3}$ increase of $\mathrm{PM}_{2.5}$ ) (Mehta et al. 2021). Meanwhile, there were discrepancies in the association between $\mathrm{PM}_{2.5}$ and $\mathrm{Hb}$. Conversely, a study revealed that $\mathrm{Hb}$ concentration increased with the rise of daily average concentration of $\mathrm{PM}_{2.5}(\beta: 9.923 \%, 95 \% \mathrm{CI}$ : $8.741 \%, 11.264 \%, \mathrm{~g} / \mathrm{L}$, per $10 \mu \mathrm{g} / \mathrm{m}^{3}$ increase of $\mathrm{PM}_{2.5}$ ) (Wang et al. 2021) and one study for pregnant women illuminated that prenatal exposure to $\mathrm{PM}_{2.5}$ linked to higher concentration of $\mathrm{Hb}(\beta: 0.929,95 \%$ CI: $0.068,1.789, \mathrm{~g} / \mathrm{L}$, per IQR increase of $\mathrm{PM}_{2.5}$ ) (Liao et al. 2019). These discrepancies might be due to the heterogeneities of population, regions, exposure periods, and constituents of $\mathrm{PM}_{2.5}$. The chronic exposure to $\mathrm{PM}_{2.5}$ might influence the $\mathrm{Hb}$ level by interfering the synthesis of $\mathrm{Hb}$, and the acute exposure to $\mathrm{PM}_{2.5}$ might accelerate the apoptosis of RBC by upregulating the gene expression of cytokines (Gao et al. 2021).

A systematic review and meta-analysis concluded that $\mathrm{BC}$ and organic carbon (OC) were the important harmful constituents of $\mathrm{PM}_{2.5}$, and the other constituents of $\mathrm{PM}_{2.5}$ also had adverse health effects (Yang et al. 2019). Although there were uncertainties, Kelly and Fussell (2015) concluded that $\mathrm{NH}_{4}{ }^{+}, \mathrm{SO}_{4}{ }^{2-}$, and $\mathrm{NO}_{3}{ }^{-}$had small threats to health. A study for normal people indicated that $\mathrm{Hb}$ was negatively correlated with $\mathrm{NH}_{4}{ }^{+}, \mathrm{NO}_{3}{ }^{-}$, and $\mathrm{SO}_{4}{ }^{2-}$ in fine particulate matter (Wang et al. 2021). An animal study elucidated that short-term exposure to diesel exhaust particles caused reduction of $\mathrm{Hb}$ concentration in rats (Nemmar and Inuwa, 2008). A study recruited 38 healthy subjects exposure to secondary organic aerosol mixed with water-soluble objects led to 8-10\% declines in RBC proteasome activity (Kipen et al. 2011). We also found that $\mathrm{BC}, \mathrm{NO}_{3}{ }^{-}$, and $\mathrm{OM}$ were tied to lower $\mathrm{Hb}$ level in this study. In addition, a handful of studies implied that prenatal exposure to $\mathrm{BC}, \mathrm{OC}, \mathrm{SO}_{4}{ }^{2-}, \mathrm{NH}_{4}{ }^{+}$, $\mathrm{NO}_{3}{ }^{-}$, and Dust were the risk factors for adverse pregnancy outcomes (Cai et al. 2020; Ebisu et al. 2018; Fong et al. 2019).

Pregnancy, breastfeeding, and monthly menstrual cycles contribute to the loss of iron in women, which lead to that multiparous women tend to have higher risk of anemia in pregnancy. A study including 426 pregnant women in Turkey discovered that the nulliparous pregnant women had higher proportion of those with normal hemoglobin values than the multiparous pregnant women (Çelik Kavak \& Kavak 2017). Al-Farsi et al. (2011) also observed that the risk ratios of anemia in pregnancy increased as the level of parity increased. During pregnancy, the plasma volume of women increases and induces hemodilution especially in the 32-34 weeks of pregnancy (Cunningham et al. 2014). The prevalence of anemia in pregnancy is affected by sociodemographic factors. The previous studies indicated that pregnant women who were ethnic minorities, with lower educational level and income had higher risk of anemia (Gebre \& Mulugeta 2015; Taner et al. 2015; Wu et al. 2020). In general, regions with high population density are coupled with more severe air pollution because of the numerous vehicles, power generation, and road dust.

$\mathrm{PM}_{2.5}$ can induce local pulmonary inflammation, and consequently evoke systemic oxidative stress and inflammation, which have been established as the main mechanism of the adverse health effects of $\mathrm{PM}_{2.5}$ (Feng et al. 2016; Hiraiwa \& van Eeden 2013). $\mathrm{PM}_{2.5}$ can induce nephrotoxicity and kidney damage, and then lower the secretion of erythropoietin (EPO) (Hsu et al. 2019; Shih et al. 2018; Zeisberg \& Kalluri 2015). The downregulated EPO undermines the proliferation and differentiation of $\mathrm{RBC}$. In addition, $\mathrm{PM}_{2.5}$ might exacerbate the erythropoietin resistance and attenuate the hematopoietic function in bone marrow (Bárány 2001; Ganz 2019). Most importantly, $\mathrm{PM}_{2.5}$ can cause immune activation and inflammatory stress, and enable the inflammatory factors and cytokines rise, such as C-reactive protein and IL-6, which have impacts on the hepcidin-ferroportin axis (D'Angelo 2013; Nemeth \& Ganz 2014). The IL-6 can upregulate hepcidin by the STAT3 signaling in hepatocytes, and the hepcidin bind with ferroportin on cell membrane, so as to induce the internalization and degradation of ferroportin, which hinders the absorption of dietary iron in duodenum, blocks the recycling of the iron from the aging RBC via iron sequestration in macrophages, and the iron might retain in the reticuloendothelial cells (Langer and Ginzburg, 2017; Weiss et al. 2019). The altered iron homeostasis finally interferes the synthesis of $\mathrm{Hb}$.

Our study had some strengths. First, we not only verified the associations of $\mathrm{PM}_{2.5}$ with $\mathrm{Hb}$ and anemia in pregnant women, but also the associations of constituents of $\mathrm{PM}_{2.5}$ with $\mathrm{Hb}$ and anemia, which added to the evidences of adverse health impacts of $\mathrm{PM}_{2.5}$ and its constituents, and helped to make targeted and effective policies. Second, the lag effects of $\mathrm{PM}_{2.5}$ and its constituents on $\mathrm{Hb}$ and anemia in pregnant women were investigated, which could aid pregnant women to figure out the sensitive window, timely use air purify equipment, and wear mask. Third, in addition to the linear associations of $\mathrm{PM}_{2.5}$ and its constituents with $\mathrm{Hb}$ and anemia, the non-linear associations were also explored, 
which could guide further studies to verify the threshold effects and drive the abatement of air pollution.

Besides, there were some limitations in this study. First, although ambient $\mathrm{PM}_{2.5}$ and its constituents are widespread and extensive, the indoor air pollution is also of important health impact on pregnant women. Ali et al. (2021) reviewed that the concentrations of pollutants were higher in indoor environment and they could increase the health risks in women and children through oxidative, DNA methylation, and gene activation. Therefore, failing to obtain the status of indoor pollution, such as household energy, cooking habits, and devices, was a limitation in this retrospective cohort study. Second, family income is related to the nutrition status and antenatal care access of pregnant women. Those with lower family income tend to have higher prevalence of anemia (Kare \& Gujo 2021), which might be ascribed to the imbalance dietary patterns and less antenatal visits missing timely intervention. Thus, in spite of the regional-level GDP and population density were adjusted in this study, which could mirror the socioeconomic status of the pregnant women to some extent, the effect of family income was still warranted to be considered. Third, dietary pattern and nutrient supplement could closely influence on the nutritional status of pregnant women. Previous studies (Abriha et al. 2014; Mayasari et al. 2021) indicated that improved meat consumption could help to mitigate the prevalence of anemia in pregnant women and intakes of carbohydrates and vegetables were correlated with gestational iron status by adjusting hepcidin levels. Hence, although gestational weight gain was included to embody the nutritional status of pregnant women, the dietary pattern and nutrient supplement were still critical confounders of the associations of $\mathrm{PM}_{2.5}$ and its constituents with $\mathrm{Hb}$ and anemia.

\section{Conclusions}

Exposure to $\mathrm{PM}_{2.5}$ and some constituents of $\mathrm{PM}_{2.5}$ was associated with reduced $\mathrm{Hb}$ level during the third trimester in multiparous pregnant women. Related departments should restrict the pirate cars, set standards for the used fossil fuel, and increase the greenness in the urban regions to alleviate the generation of $\mathrm{PM}_{2.5}$ and its constituents. Pregnant women should use air purify and put masks when the air pollution is severe to mitigate the inhalation of $\mathrm{PM}_{2.5}$ and its constituents, ultimately eliminate their detrimental effects.

Supplementary Information The online version contains supplementary material available at https://doi.org/10.1007/s11356-022-18693-2.

Acknowledgements Thanks for all participants, staff of the First Affiliated Hospital of Xi' an Jiaotong University, the Atmospheric Composition Analysis Group, Institute of Geographic Sciences and Natural Resources Research, Chinese Academy of Sciences, and WorldPop.
Author contribution GX, JY, and WY designed the study. GX and JY conducted the data analysis and wrote the manuscript. LY, MX, LS, BZ, LG, and MCC collected and integrated the data. All authors have been involved in drafting the manuscript or revising it critically for important intellectual content; they have given final approval of the version to be published.

Funding This study was supported by the National Social Science Foundation of China (Program No. 20BRK037). The funders did not participate in any part of the study from design to the writing of the manuscript, except for supporting this project.

Availability of data and materials The data was available from the corresponding author on reasonable request.

\section{Declarations}

Ethics approval and consent to participate The consent to participate was waivered due to this study analyzed with de-identified data, and this study was approved by the Medical Ethics Committee of the First Affiliated Hospital of Xi'an Jiaotong University (No. XJTU1AF2020LSK-261) based on the institution's ethical standards and the ethical standards of the Declaration of Helsinki.

Consent for publication Not applicable.

Competing interests The authors declare no competing interests.

Open Access This article is licensed under a Creative Commons Attribution 4.0 International License, which permits use, sharing, adaptation, distribution and reproduction in any medium or format, as long as you give appropriate credit to the original author(s) and the source, provide a link to the Creative Commons licence, and indicate if changes were made. The images or other third party material in this article are included in the article's Creative Commons licence, unless indicated otherwise in a credit line to the material. If material is not included in the article's Creative Commons licence and your intended use is not permitted by statutory regulation or exceeds the permitted use, you will need to obtain permission directly from the copyright holder. To view a copy of this licence, visit http://creativecommons.org/licenses/by/4.0/.

\section{References}

Abriha A, Yesuf ME, Wassie MM (2014) Prevalence and associated factors of anemia among pregnant women of Mekelle town: a cross sectional study. BMC Res Notes 7:888. https://doi.org/10. 1186/1756-0500-7-888

Al-Farsi YM, Brooks DR, Werler MM, Cabral HJ, Al-Shafei MA, Wallenburg HC (2011) Effect of high parity on occurrence of anemia in pregnancy: a cohort study. BMC Pregnancy Childbirth 11:7. https://doi.org/10.1186/1471-2393-11-7

Ali SA, Tikmani SS, Saleem S, Patel AB, Hibberd PL, Goudar SS, Dhaded S, Derman RJ, Moore JL, McClure EM, Goldenberg RL (2020) Hemoglobin concentrations and adverse birth outcomes in South Asian pregnant women: findings from a prospective Maternal and Neonatal Health Registry. Reprod Health 17:154. https:// doi.org/10.1186/s12978-020-01006-6

Ali MU, Yu Y, Yousaf B, Munir MAM, Ullah S, Zheng C, Kuang X, Wong MH (2021) Health impacts of indoor air pollution from household solid fuel on children and women. J Hazard Mater 416:126127. https://doi.org/10.1016/j.jhazmat.2021.126127 
Badman DG, Jaffé ER (1996) Blood and air pollution: state of knowledge and research needs. Otolaryngol Head Neck Surg 114:205208. https://doi.org/10.1016/s0194-5998(96)70166-3

Bárány P (2001) Inflammation, serum C-reactive protein, and erythropoietin resistance. Nephrol Dial Transplant 16:224-227. https:// doi.org/10.1093/ndt/16.2.224

Cai J, Zhao Y, Kan J, Chen R, Martin R, van Donkelaar A, Ao J, Zhang J, Kan H, Hua J (2020) Prenatal exposure to specific PM2.5 chemical constituents and preterm birth in China: a nationwide cohort study. Environ Sci Technol 54:14494-14501. https://doi. org/10.1021/acs.est.0c02373

Çelik Kavak E, Kavak SB (2017) The association between anemia prevalence, maternal age and parity in term pregnancies in our city. Perinatal Journal 25:6-10

Cunningham FG, Leveno KJ, Bloom SL, Spong CY, Dashe JS, Hoffman BL (2014) Williams obstetrics. McGraw-Hill Education, New York

D'Angelo G (2013) Role of hepcidin in the pathophysiology and diagnosis of anemia. Blood Res 48:10-15. https://doi.org/10.5045/ br.2013.48.1.10

Ebisu K, Malig B, Hasheminassab S, Sioutas C, Basu R (2018) Cause-specific stillbirth and exposure to chemical constituents and sources of fine particulate matter. Environ Res 160:358-364. https://doi.org/10.1016/j.envres.2017.10.015

Elbarbary M, Honda T, Morgan G, Guo Y, Guo Y, Kowal P, Negin J (2020) Ambient air pollution exposure association with anaemia prevalence and haemoglobin levels in Chinese older adults. Int $\mathbf{J}$ Environ Res Public Health 1710.3390/ijerph17093209

Feng S, Gao D, Liao F, Zhou F, Wang X (2016) The health effects of ambient PM2.5 and potential mechanisms. Ecotoxicol Environ Saf 128:67-74. https://doi.org/10.1016/j.ecoenv.2016.01.030

Fong KC, Di Q, Kloog I, Laden F, Coull BA, Koutrakis P, Schwartz JD (2019) Relative toxicities of major particulate matter constituents on birthweight in Massachusetts. Environ Epidemiol 3:e047. https://doi.org/10.1097/ee9.0000000000000047

Forman EJ, Treff NR, Scott RT Jr (2011) Fertility after age 45: from natural conception to assisted reproductive technology and beyond. Maturitas 70:216-221. https://doi.org/10.1016/j.matur itas.2011.07.003

Ganz T (2019) Anemia of inflammation. N Engl J Med 381:11481157. https://doi.org/10.1056/NEJMra1804281

Gao K, Chen X, Zhang L, Yao Y, Chen W, Zhang H, Han Y, Xue T, Wang J, Lu L, Zheng M, Qiu X, Zhu T (2021) Associations between differences in anemia-related blood cell parameters and short-term exposure to ambient particle pollutants in middleaged and elderly residents in Beijing, China. Sci Total Environ, 151520.https://doi.org/10.1016/j.scitotenv.2021.151520

Gebre A, Mulugeta A (2015) Prevalence of anemia and associated factors among pregnant women in north western zone of Tigray, northern Ethiopia: a cross-sectional study. J Nutr Metab 2015:165430. https://doi.org/10.1155/2015/165430

Ghassabian A et al (2019) Association of exposure to ambient air pollution with thyroid function during pregnancy. JAMA Netw Open 2:e1912902. https://doi.org/10.1001/jamanetworkopen. 2019.12902

Gluckman PD, Buklijas T, Hanson MA (2016) Chapter 1 - the developmental origins of health and disease $(\mathrm{DOHaD})$ concept: past, present, and future. In: Rosenfeld CS (ed) The epigenome and developmental origins of health and disease. Academic Press, Boston, pp 1-15

Hammer MS, van Donkelaar A, Li C, Lyapustin A, Sayer AM, Hsu NC, Levy RC, Garay MJ, Kalashnikova OV, Kahn RA, Brauer M, Apte JS, Henze DK, Zhang L, Zhang Q, Ford B, Pierce JR, Martin RV (2020) Global estimates and long-term trends of fine particulate matter concentrations (1998-2018). Environ Sci Technol 54:7879-7890. https://doi.org/10.1021/acs.est.0c01764
Hare-Mustin RT (1982) China's Marriage Law: a model for family responsibilities and relationships. Fam Process 21:477-481. https://doi.org/10.1111/j.1545-5300.1982.00477.x

Hiraiwa K, van Eeden SF (2013) Contribution of lung macrophages to the inflammatory responses induced by exposure to air pollutants. Mediators Inflamm 2013:619523. https://doi.org/10.1155/ 2013/619523

Honda T, Pun VC, Manjourides J, Suh H (2017) Anemia prevalence and hemoglobin levels are associated with long-term exposure to air pollution in an older population. Environ Int 101:125-132. https://doi.org/10.1016/j.envint.2017.01.017

Hsu YH, Chuang HC, Lee YH, Lin YF, Chen YJ, Hsiao TC, Wu MY, Chiu HW (2019) Traffic-related particulate matter exposure induces nephrotoxicity in vitro and in vivo. Free Radic Biol Med 135:235-244. https://doi.org/10.1016/j.freeradbiomed.2019.03. 008

Jung J, Rahman MM, Rahman MS, Swe KT, Islam MR, Rahman MO, Akter S (2019) Effects of hemoglobin levels during pregnancy on adverse maternal and infant outcomes: a systematic review and meta-analysis. Ann N Y Acad Sci 1450:69-82. https://doi.org/ $10.1111 /$ nyas. 14112

Kare AP, Gujo AB (2021) Anemia among pregnant women attending ante natal care clinic in Adare General Hospital, southern Ethiopia: prevalence and associated factors. Health Serv Insights 14:11786329211036304. https://doi.org/10.1177/1178632921 1036303

Kelly FJ, Fussell JC (2012) Size, source and chemical composition as determinants of toxicity attributable to ambient particulate matter. Atmos Environ 60:504-526. https://doi.org/10.1016/j.atmos env.2012.06.039

Kelly FJ, Fussell JC (2015) Air pollution and public health: emerging hazards and improved understanding of risk. Environ Geochem Health 37:631-649. https://doi.org/10.1007/s10653-015-9720-1

Kipen HM, Gandhi S, Rich DQ, Ohman-Strickland P, Laumbach R, Fan ZH, Chen L, Laskin DL, Zhang J, Madura K (2011) Acute decreases in proteasome pathway activity after inhalation of fresh diesel exhaust or secondary organic aerosol. Environ Health Perspect 119:658-663. https://doi.org/10.1289/ehp.1002784

Langer AL, Ginzburg YZ (2017) Role of hepcidin-ferroportin axis in the pathophysiology, diagnosis, and treatment of anemia of chronic inflammation. Hemodial Int 21(Suppl 1):S37-s46. https:// doi.org/10.1111/hdi.12543

Liao J, Li Y, Wang X, Zhang B, Xia W, Peng Y, Zhang W, Cao Z, Zhang Y, Liang S, Hu K, Xu S (2019) Prenatal exposure to fine particulate matter, maternal hemoglobin concentration, and fetal growth during early pregnancy: associations and mediation effects analysis. Environ Res 173:366-372. https://doi.org/10.1016/j. envres.2019.03.056

Lin L, Li Q, Yang J, Han N, Chen G, Jin C, Xu X, Liu Z, Liu J, Luo S, Raat H, Guo Y, Wang H (2020) The associations of residential greenness with fetal growth in utero and birth weight: a birth cohort study in Beijing. China Environ Int 141:105793. https:// doi.org/10.1016/j.envint.2020.105793

Mayasari NR, Bai CH, Hu TY, Chao JC, Chen YC, Huang YL, Wang FF, Tinkov AA, Skalny AV, Chang JS (2021) Associations of food and nutrient intake with serum hepcidin and the risk of gestational iron-deficiency anemia among pregnant women: a populationbased study. Nutrients 1310.3390/nu13103501

Mehta U, Dey S, Chowdhury S, Ghosh S, Hart JE, Kurpad A (2021) The association between ambient PM2.5 exposure and anemia outcomes among children under five years of age in India. Environ Epidemiol 5:e125. https://doi.org/10.1097/ee9.0000000000 000125

Morales-Ancajima VC, Tapia V, Vu BN, Liu Y, Alarcón-Yaquetto DE, Gonzales GF (2019) Increased outdoor PM2.5 concentration is associated with moderate/severe anemia in children aged 6-59 
months in Lima, Peru. J Environ Public Health 2019, 6127845. https://doi.org/10.1155/2019/6127845

Nemeth E, Ganz T (2014) Anemia of inflammation. Hematol Oncol Clin North Am 28(671-81):vi. https://doi.org/10.1016/j.hoc.2014. 04.005

Nemmar A, Inuwa IM (2008) Diesel exhaust particles in blood trigger systemic and pulmonary morphological alterations. Toxicol Lett 176:20-30. https://doi.org/10.1016/j.toxlet.2007.09.006

Seaton A, Soutar A, Crawford V, Elton R, McNerlan S, Cherrie J, Watt M, Agius R, Stout R (1999) Particulate air pollution and the blood. Thorax 54:1027-1032. https://doi.org/10.1136/thx.54. 11.1027

Shang L, Huang L, Yang W, Qi C, Yang L, Xin J, Wang S, Li D, Wang B, Zeng L, Chung MC (2019) Maternal exposure to PM2.5 may increase the risk of congenital hypothyroidism in the offspring: a national database based study in China. BMC Public Health 19:1412. https://doi.org/10.1186/s12889-019-7790-1

Shih HM, Wu CJ, Lin SL (2018) Physiology and pathophysiology of renal erythropoietin-producing cells. J Formos Med Assoc 117:955-963. https://doi.org/10.1016/j.jfma.2018.03.017

Taner CE, Ekin A, Solmaz U, Gezer C, Çetin B, Keleşoğlu M, Erpala MB, Özeren M (2015) Prevalence and risk factors of anemia among pregnant women attending a high-volume tertiary care center for delivery. J Turk Ger Gynecol Assoc 16:231-236. https:// doi.org/10.5152/jtgga.2015.15071

Ullah A, Sohaib M, Saeed F, Iqbal S (2019) Prevalence of anemia and associated risk factors among pregnant women in Lahore, Pakistan. Women Health 59:660-671. https://doi.org/10.1080/03630 242.2018.1544966

van Donkelaar A, Martin RV, Li C, Burnett RT (2019) Regional estimates of chemical composition of fine particulate matter using a combined geoscience-statistical method with information from satellites, models, and monitors. Environ Sci Technol 53:25952611. https://doi.org/10.1021/acs.est.8b06392

van Donkelaar A, Hammer MS, Bindle L, Brauer M, Brook JR, Garay MJ, Hsu NC, Kalashnikova OV, Kahn RA, Lee C, Levy RC, Lyapustin A, Sayer AM, Martin RV (2021) Monthly global estimates of fine particulate matter and their uncertainty. Environ Sci Technol 55:15287-15300. https://doi.org/10.1021/acs.est.1c05309
Wang S, Kaur M, Li T, Pan F (2021) Effect of different pollution parameters and chemical components of PM2.5 on health of residents of Xinxiang City, China. Int J Environ Res Public Health 1810.3390/ijerph18136821

Weiss G, Ganz T, Goodnough LT (2019) Anemia of inflammation. Blood 133:40-50. https://doi.org/10.1182/blood-2018-06-856500

WHO (2011) Haemoglobin concentrations for the diagnosis of anaemia and assessment of severity. World Health Organization, Geneva

WHO (2015) The global prevalence of anaemia in 2011. World Health Organization, Geneva

Wu Y, Ye H, Liu J, Ma Q, Yuan Y, Pang Q, Liu J, Kong C, Liu M (2020) Prevalence of anemia and sociodemographic characteristics among pregnant and non-pregnant women in southwest China: a longitudinal observational study. BMC Pregnancy Childbirth 20:535. https://doi.org/10.1186/s12884-020-03222-1

Xie G, Sun L, Yang W, Wang R, Shang L, Yang L, Qi C, Xin J, Yue J, Chung MC (2021) Maternal exposure to PM2.5 was linked to elevated risk of stillbirth. Chemosphere 283:131169. https://doi. org/10.1016/j.chemosphere.2021.131169

Yang Y, Ruan Z, Wang X, Yang Y, Mason TG, Lin H, Tian L (2019) Short-term and long-term exposures to fine particulate matter constituents and health: a systematic review and meta-analysis. Environ Pollut 247:874-882. https://doi.org/10.1016/j.envpol. 2018.12.060

Young MF, Oaks BM, Tandon S, Martorell R, Dewey KG, Wendt AS (2019) Maternal hemoglobin concentrations across pregnancy and maternal and child health: a systematic review and meta-analysis. Ann N Y Acad Sci 1450:47-68. https://doi.org/10.1111/nyas. 14093

Zeisberg M, Kalluri R (2015) Physiology of the renal interstitium. Clin J Am Soc Nephrol 10:1831-1840. https://doi.org/10.2215/ cjn.00640114

Zhao Y, Cai J, Zhu X, van Donkelaar A, Martin RV, Hua J, Kan H (2020) Fine particulate matter exposure and renal function: a population-based study among pregnant women in China. Environ Int 141:105805. https://doi.org/10.1016/j.envint.2020.105805

Publisher's Note Springer Nature remains neutral with regard to jurisdictional claims in published maps and institutional affiliations. 